\title{
Automated Sewer Pipe Inspection through Image Processing
}

\author{
Olga Duran, Kaspar Althoefer, Lakmal D. Seneviratne \\ Department of Mechanical Engineering \\ King's College, Strand, London WC2R 2LS, UK \\ email: olga.duran@kcl.ac.uk (k.althoefer@kcl.ac.uk)
}

\begin{abstract}
An innovative inspection method to assess the condition of sewer pipes is proposed in this paper. The standard sewer inspection technique, based on closed-circuit television systems, has a very poor performance. A video camera is mounted on a robot and the video recording is provided off-line to an engineer that classifies the potential defects. The focus of the research is the automated identification and location of discontinuities in the internal surface of sewers. The transducer used is an assembly of a CCD camera and optics elements to generate a ring-shaped laser pattern. The automated inspection method consists of several stages including the segmentation of the image into characteristic geometric features and potential defect regions. Automatic recognition, rating and classification of pipe defects are carried out by means of computation of a partial histogram based on adaptive image processing. Experiments in a realistic environment have been conducted and results are presented.
\end{abstract}

\section{Introduction}

It is estimated that, mainly due to their age, around $20 \%$ of sewer networks are damaged [1]. According to a study by an UK-based company, there are approximately 5,000 collapses and 200,000 blockages per year in the United Kingdom [2]. Early detection of pipe faults may prevent severe failures that can involve environmental damage and high economic costs.

A large part of pipes used in municipal sewer networks is circular with an inner diameter between 0.1 and $1 \mathrm{~m}$. Humans cannot access these pipes directly. Remotely controlled inspection devices based on mobile robots or wheeled platforms equipped with sensors are used instead. The standard approach is to have a closed circuit television (CCTV) camera fitted on a mobile platform that travels through the pipe recording images onto a videotape. During inspection the operator uses the feedback camera signals to steer the vehicle. The recorded images are assessed off-line by an engineer. This method has some disadvantages such as being slow, expensive, prone to human errors and allowing the inspection of nonflooded pipes only. Considering that typically, the lowest water level in a sewer is around 5\%, in a combined sewer during dry weather, other sensors are needed to inspect the flooded part of the pipe. Moreover, the amount of information generated (about 30 hours of video for the inspection of $10 \mathrm{Km}$ of line) is exorbitant and a major hindrance for conducting large-scale surveys. The time required to inspect the pipe depends also on the number of defects, since at each defect the operator must stop, assess, and record the condition. Thus, the inspection of a heavily damaged pipe section will take much longer than a fairly intact pipe section [3]. Therefore, it is difficult to estimate time and cost to perform CCTV surveys. Owing to the variability and the time consumption incurred by the human based assessment process, automation of this part of the inspection task becomes an important issue.

The investigation reported in this paper is part of a research program that aims to develop automated sewer inspection methods. The objective of this project is to study and develop multi-sensor systems and to create intelligent sensor fusion and sensor data processing algorithms. It is envisaged that a laser-profiler and CCD camera would measure the surface geometry of the drained part of a sewer, while a sonar scanner measures the flooded part. This paper presents research progress on the inspection and condition assessment of the nonflooded part of the pipe, using a structured light source and a ring-pattern projector.

Research into sewer inspection using camera-based profiling techniques is still in its infancy. However, some promising work is currently being carried out at different research institutes. The pipe profiling tool developed and studied by Thames Water and OMC (UK-based companies) is an example of the single spot method, where a mechanism incorporating a light-sensitive sensor and a laser rotates continuously in order to measure the size and shape of pipes. The KARO robot, a development by the Fraunhofer-Institut für lnformations- und Datenverarbeitung IITB Karlsruhe [4], is a system where a camera analyses rings of light projected onto the internal pipe wall, allowing the detection of pipe deformations and obstacles. The Department of Civil Engineering at the University of Wales (UK) have a special light head that attached to the camera of a normal CCTV platform can allow the measurement of pipe 
profiles and consequently the detection of changes in the sewer shape [5]. All those examples are only able to detect deformation of the pipe. One important disadvantage of existing methods is that they have difficulties in recognising structural failures such as cracks. The proposed method provides novel algorithms to overcome this problem, by identifying and locating such structural failures using the intensity distribution along the projected ring in a computationally efficient way. Furthermore, the method described makes use of an intelligent classification stage, which provides clear identification of defective pipe segments. The different processing stages such as data acquisition, image processing and classification are described in the following Sections. In Section 2, the transducer used to acquire the images is described. The segmentation of the image into regions of interest will be shown in Section 3. The feature extraction step that extracts and analyses the information from the segmented images is shown in Section 4. Finally, conclusions are given in Section 5.

\section{CCTV-based laser profiler}

The CCTV-based system consists of an optical pattern generator that projects rings of laser light onto the wall, illuminating a pipe section. The optical ring generator is made of an assembly of a laser generator and diffractive diffuser optics. Depending on the diffuser optics used, the reflected light forms either one or several concentric cones of light with a specific projection angle and width (fig. 1).

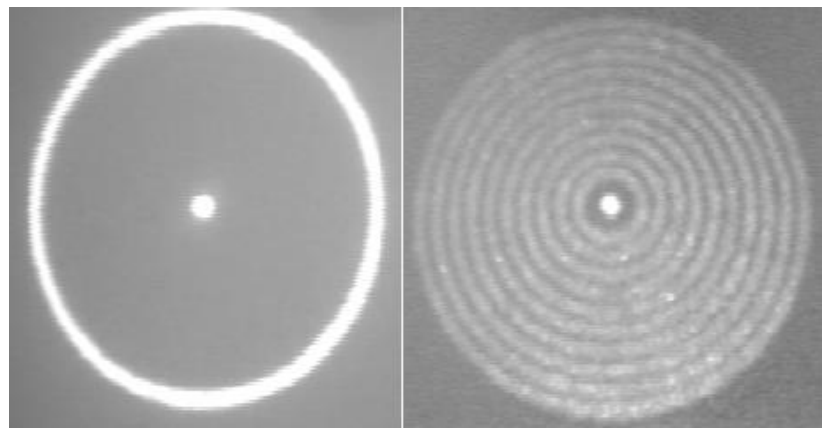

(a)

(b)

Fig 1: Projected rings of light:

a) a single ring diffuser is used;

b) 24 concentric-rings diffuser is used

The experimental rig used consists of a $1 \mathrm{~mW}$ semiconductor laser diode of $635 \mathrm{~nm}$ wavelength, a diffractive diffuser with a ring pattern (fig. 2).

The projected circular patterns of light are read by a calibrated CCD camera, and stored in a computer via a frame grabber (fig. 3). The tests were conducted on PVC pipe sections with inner diameters of 260 and $300 \mathrm{~mm}$. Holes were pierced in the pipe segments in order to simulate defects.

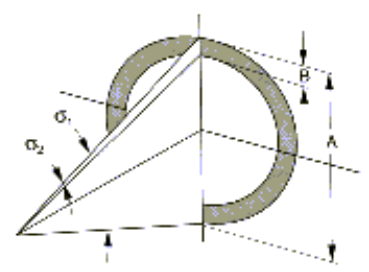

Fig 2: Diffractive diffuser geometric characteristics: In our set-up, the diffractive diffuser beam angles were $\sigma_{1}$ $=10.5^{\circ}, \sigma_{2}=0.2^{\circ}$

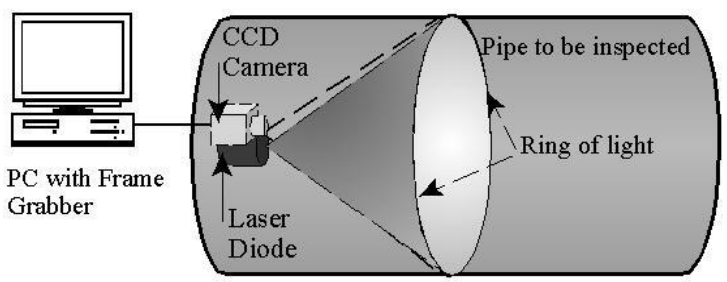

Fig 3: Experimental set-up, where a camera and a laser projector are placed next to each other. The camera images are read into a PC by means of a frame-grabber.

Pipe cracks and local discontinuities can be considered as distinct transients in an otherwise non-defective pipe surface. Such transients can be detected by analysing the light intensity of the projected rings. In this study, circles of laser light are projected onto the pipe wall and are reflected back onto the CCD detector inside the camera. At points where discontinuities occur, the laser light is scattered resulting in changes of intensity levels in the acquired camera image (fig. 4). By analysing these intensity levels, defects can be detected and located.

The proposed profiling assembly has many advantages over traditional CCTV systems:

a) There is no need for a reference level of intensity or luminosity as the information is treated as differential.

b) An entire profile or multiple profiles can be inspected in one go. Here, the single profile approach is presented.

c) Deflections, discontinuities and cracks can be identified.

d) As the geometric characteristics of the cone of light are fixed for a given diffuser optic, the location of the surface under investigation relative to the platform is known. 
e) The proposed method can easily be incorporated into existing camera-based systems.

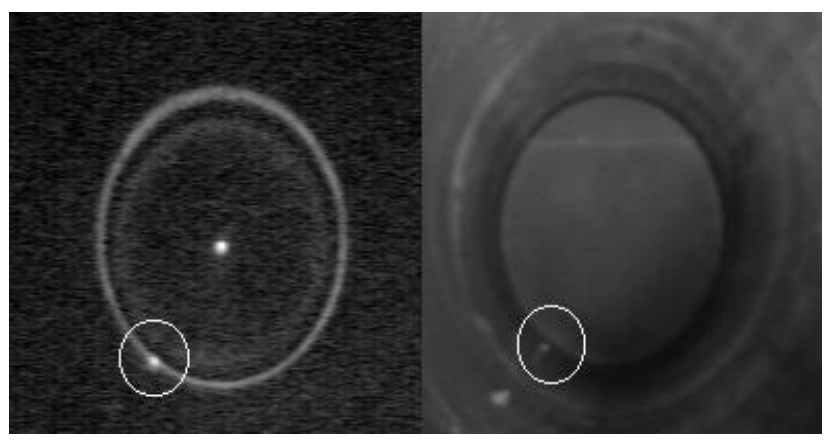

(a)

(b)

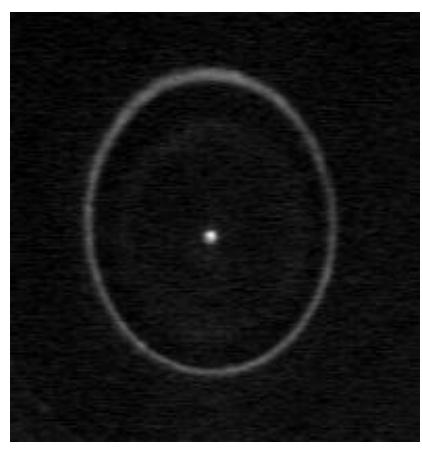

(c)

Fig 4: Images of a pipe with a $2 \mathrm{~mm}$ hole. The location of the hole is marked with a small circle.

a) Image acquired using the laser-based transducer, where the crack can be easily identified.

b) Image acquired with a conventional CCD camera and lighting system. Note that the white spot next to the circle is created by a light reflection - it is not a defect - and could be identified as a fault.

c) Image acquired using the laser-based transducer, corresponding to the location of the non-defective point caused by light reflection as referred to under b) of this figure. This image shows that the proposed method correctly presents this point as non-defective.

\section{Image Segmentation}

This section describes the various image-processing stages to identify the location and specific shape of the ring profile and to separate it from the image background. After the extraction of the ring profile, defects can be searched for along the profile. This approach, instead of searching the entire image for potentially defective areas, considerably reduces the volume of data to be processed and consequently the computation time. The projection of a circle into the camera is often an ellipse. Moreover, any misalignment of the projector device with respect to the pipe centre will cause the ring projection to degenerate into an ellipse. Clustering methods such as Hough transform have been tried for fitting the elliptic image acquired by the camera to a conic equation. Besides some advantages, such as high robustness to occlusions and lack of pre-segmentation requirements, they are computationally complex and do provide multiple solutions. In this work, a highly efficient specific ellipsefitting algorithm is used. A signal conditioning stage including filtering precedes the conic fitting stage, in order to enhance the quality of the images and ease the identification of the ellipse [6].

\subsection{Signal conditioning}

Image processing methods are applied in order to enhance image quality, prior to applying the conic-fitting algorithm. Two filtering steps are applied, followed by an edge detector [6].

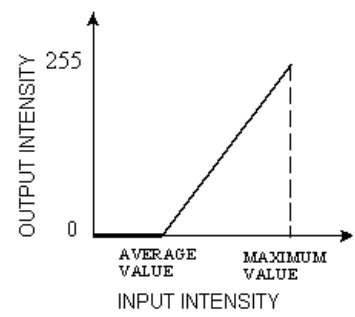

(a)

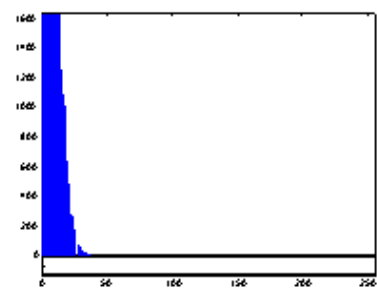

Irquit image histogram

(b)

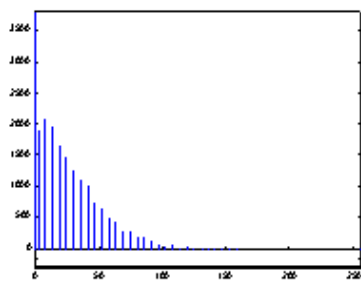

Output image histogram

(c)
Fig. 5: Adjustment of intensity levels: a) intensity computation, b) histogram before adjustment, c) histogram after adjustment.

In order to increase the picture contrast, the intensity levels of all the image pixels are adjusted to span the entire available intensity range $(0,255)$. This is an adaptive image processing step where the pixels intensities are mapped in such a way that all input intensities that fall below the average intensity value 
(background) are mapped to zero intensity, and all intensities higher than the average are "expanded" to span the entire image intensity range (Fig. 5).

This procedure brightens the image considerably, and also widens the dynamic range of the dark portions of the original image, making it much easier to identify the circle of light. Subsequently, a median filter that simultaneously reduces noise and preserves edges is applied. Finally, a canny edge detector has been found most efficient to pre-segment the image by finding the edges of the ring of light [6].

\subsection{Ellipse extraction - conic fitting}

In this work, the ellipse fitting method proposed by Fitzgibbon [7], improved by a method suggested by Halýr [8], is used. The approach is a non-iterative algorithm based on a least squares minimisation of the algebraic distance between the data points and the ellipse. A quadratic constraint guarantees an ellipse-specific solution even for scattered or noisy data, or for a partial occlusion condition, which can occur when the sewer is partially flooded.

A conic can be represented by an implicit second order polynomial:

$$
\begin{aligned}
& F(\bar{a}, \bar{x})=\bar{a} \cdot \bar{x}= \\
& =a \cdot x^{2}+b \cdot x \cdot y+c \cdot y^{2}+d \cdot x+e \cdot y+f=0
\end{aligned}
$$

$$
\text { Where, } \begin{aligned}
\bar{a} & =\left[\begin{array}{llllll}
a & b & c & d & e & f
\end{array}\right]^{T} \\
\bar{x} & =\left[\begin{array}{llllll}
x^{2} & x \cdot y & y^{2} & x & y & 1
\end{array}\right]^{T}
\end{aligned}
$$

and $F(\bar{a}, \bar{x})$ is the algebraic distance of a point (x, y) to the conic $F(\bar{a}, \bar{x})=0$.

The fitting consists of minimising the squares of the distances: $\sum_{i=1}^{N} F\left(\bar{x}_{i}\right)^{2}$ subject to the equality constraint $4 a c-b^{2}=1$ that incoporates both scaling and ellipsespecific conditions.

To improve the behaviour of the algorithm in the presence of noise, especially when points that do not correspond to the projected ring appear, a weighted function is applied to the points extracted from the image after edge detection, before feeding them to the above algorithm. The points are weighted with a function of an average distance measure between the data points and the centre of the projected ring. The function ensures that all weights are in the range [0 1] (fig. 6).

\section{Feature extraction}

The objective of the feature extraction stage is to extract and analyse useful information from the segmented image. On the one hand, an appropriate representation of the data contained in the image is achieved, and on the other hand the complexity of this data is considerably reduced. Firstly, a partial histogram of the image is created by computing local averages of intensity along the ring profile. Subsequently, faults can be identified and located analysing successive histograms.

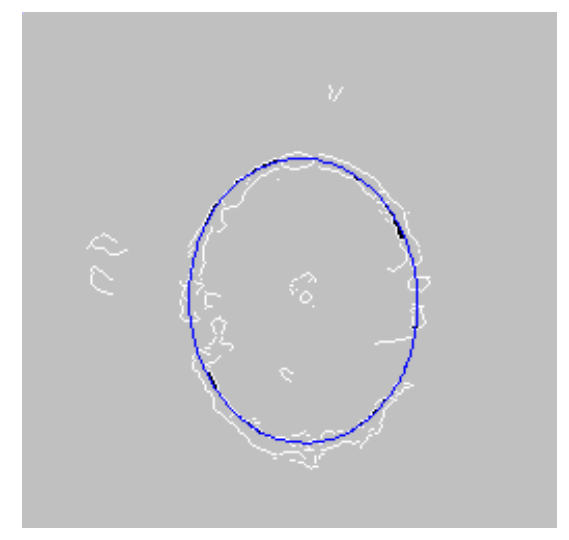

Fig 6: Ellipse fitting with real noisy data acquired with a CCD camera and a single-ring laser profiler.

\subsection{Partial histogram}

The feature extraction algorithm computes local averages of intensity along a defined number of segments of the ellipse. The local average intensity is computed using the formula: $\frac{\sum \mu \cdot x}{\sum \mu}$, where $\mathrm{x}$ is the grey level of a certain image point, and $\mu$ the frequency of that grey level [9].

Since the image of the ring of light is wider than one pixel, the average is computed over a rectangle covering individual ellipse segments. The size of that rectangle has been fixed, taking into account the geometric characteristics of the projected cone of light and the displacement step along the pipe (fig. 2).

Figure 7 shows the results of the feature extraction process displayed as a partial image histogram. In the current experiment, a pipe of $260 \mathrm{~mm}$ of diameter is used. A $2 \mathrm{~mm}$ crack has been pierced at 122 degrees from the horizontal axis and at $4 \mathrm{~cm}$ from the original position of the transducer. 
The partial histograms have been computed with steps of one degree along the ellipse segments. In order to get a full representation of the inner walls of the pipe, successive histograms are computed from the acquired pipe surface images which are taken while the platform travels along the pipe. Here, steps of $1 \mathrm{~cm}$ in the longitudinal direction are taken.

With consecutive histograms an open-up representation of the pipe surface is created, where abrupt peaks of intensity in radial as well as longitudinal directions show the location of faults (fig.8). Figure 9 shows a histogram where the intensity changes are only present in the radial direction but not in the longitudinal. This histogram represents a variation of the lighting condition in the longitudinal direction, but not a defect.

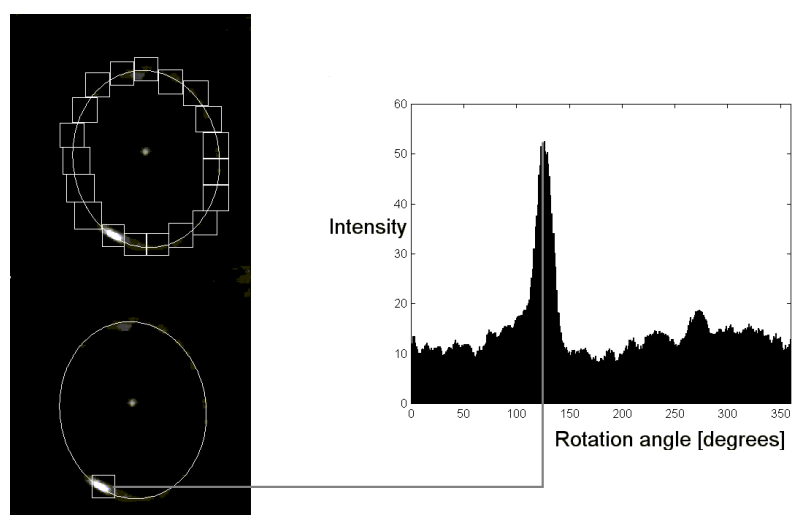

Fig 7: Windowing the image along the ellipse. The peak indicates the location of a potential discontinuity.

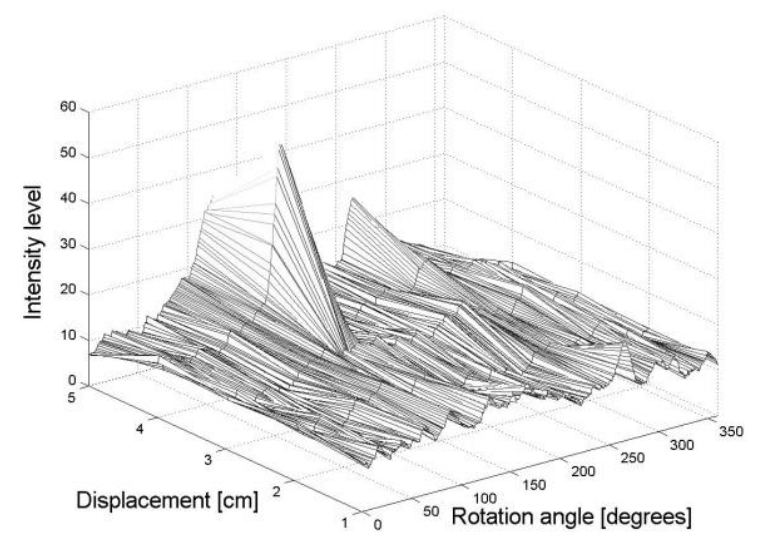

Fig 8: Open-up representation of the inner surface of the pipe. The peak intensity shows the location of a potential defective section.

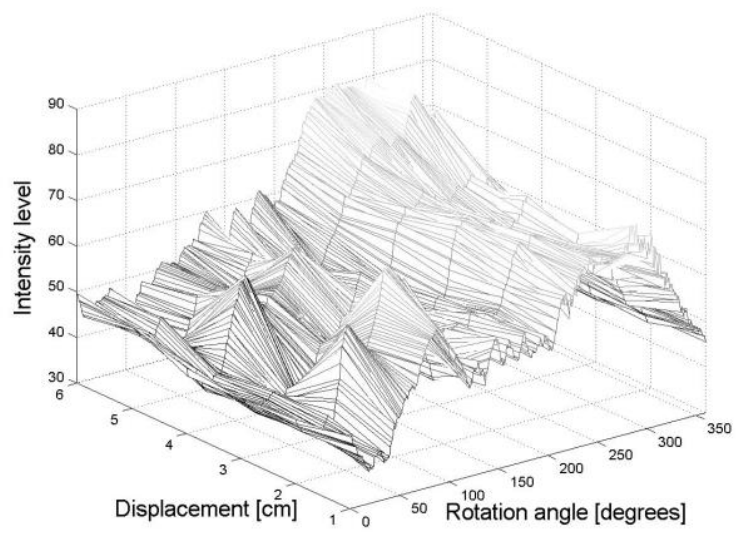

Fig 9: Open-up representations of the inner surface of the pipe. The change of intensity in the radial direction shows a variation in the lighting condition.

\subsection{Fault location}

In this work, a gradient method is applied in order to extract defect regions from the pipe surface image. The main interest here is to identify the points where the variation in intensity levels is high, regardless of the lighting conditions. Note that in a typical pipe fault, the edges of the crack will be located when using this approach. . The computation of the gradient of the surface image $f(d, \alpha)$ is based on obtaining the partial derivatives $\frac{\partial f}{\partial d}$ and $\frac{\partial f}{\partial \alpha}$, with $d$ being the displacement along the pipe in the longitudinal direction and $\alpha$ being the rotation angle along a pipe profile [6]. If both partial derivatives at a certain point are greater than specific thresholds, a possible faulty area is identified. The optimal thresholds are calculated using the frequency of each grey level, as a function of the average grey level and the grey level corresponding to the maximum frequency. These thresholds are recalculated and applied to every row of data. A row of data for the derivative with respect to the rotation angle is considered as an entire profile at a fixed displacement point. And the displacement points at a fixed rotation angle are taken for the derivative with respect to the longitudinal axis calculations. In fig. 10 and 11, results showing the location of a fault when using this approach are shown together with the partial derivatives at this fault location. 


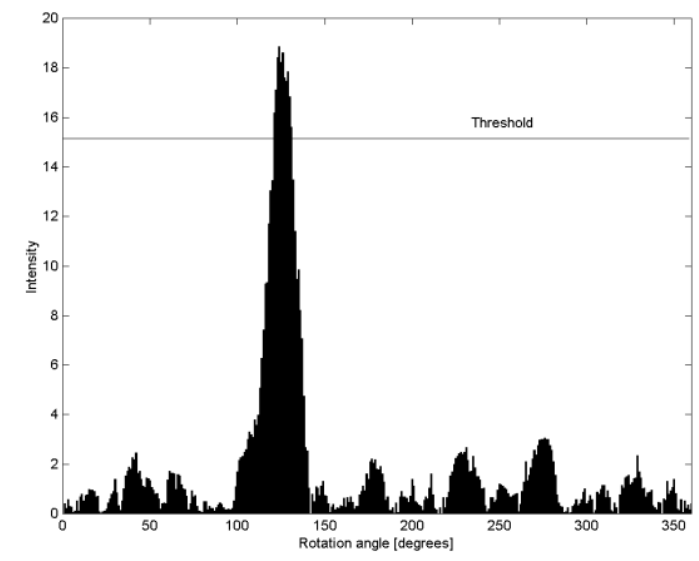

Fig 10: Partial derivative $\partial f / \partial \alpha$ at a fault location point, with applied threshold (14.78).

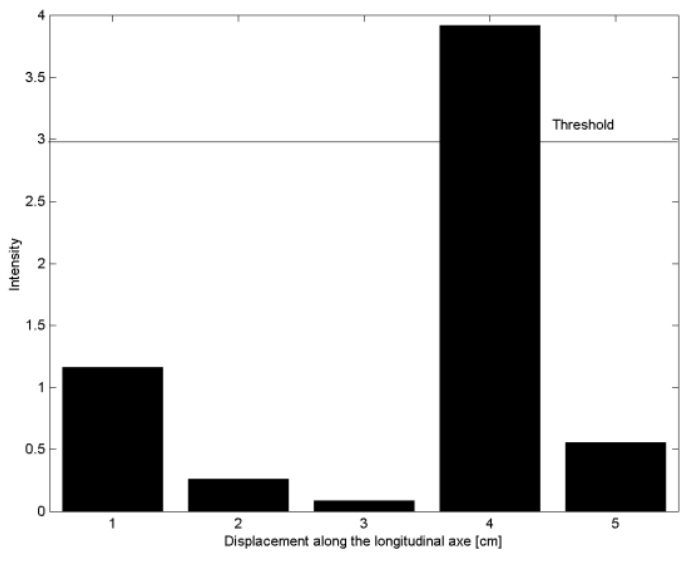

Fig 11: Partial derivative $\partial f / \partial d$ at the same fault location point, with applied threshold (2.99).

\section{Conclusions}

A new laser-based inspection system to assess the condition of sewer pipes is presented. The method is based on the projection of a laser-generated pattern onto the pipe walls, allowing the location of cracks in the inner surface of pipes by analysing the light intensity of the projected rings. Tests have been conducted in plastic pipes with different inner diameters, where cracks up to 2 $\mathrm{mm}$ have been located. This laser profiler as well as the suggested defect detection method can be easily incorporated to existing CCTV systems, improving greatly their behaviour, especially in the lack of visibility. Moreover, as the information is treated in a differential way, there is no need for a reference point.

The long-term objective of this research is to assess partially flooded pipes using a multi-sensor system. The transducer described in this paper inspects the nonflooded surface, while an ultrasonic sensor will inspect the flooded parts. In order to get the condition of the entire surface of the pipe, sensor fusion can be done at the pixel or feature level. Moreover, the information acquired by the ultrasonic sensor will be used in order to improve the behaviour of the method described in the present paper, especially against noise and under conditions where the camera images is partially occluded.

\section{Bibliography}

[1] Roth, H, Schilling, K. Navigation and Control for Pipe Inspection and Repair Robots: Proc of IFAC World Congress, 1999.

[2] WEF Manuals \& Reports FD-6, ASCE Manuals \& reports on engineering, Existing sewer evaluation and rehabilitation, No. 62, 1994.

[3] Pace, NG. Ultrasonic surveying of fully charged sewage pipes, Electronics and Communications Engineering Journal, 1994, pp 87-92.

[4] Kuntze, H-B. Schmitdt, D. Haffner, H. Loh, M. KARO, a flexible robot for smart sensor-based sewer inspection, International No Dig'95, Dresden, 1995.

[5] Henry, R. Luxmoore, AR. A pipe-profiling adapter for CCTV inspection cameras: Development of a pipeprofiling instrument. Measurement Science and Technology, Vol. 7, 1996, pp 495-504.

[6] Gonzalez R.C. Digital Image Processing. AddisonWesley, MA, 1987.

[7] Fitzgibbon, A. Pilu, M. Fisher R. Direct Least Square Fitting of Ellipses. Pattern analysis and machine intelligence, 1999, Vol. 21 Issue 5, pp 476-480.

[8] Halir, R. Flusser J.: Numerically stable direct least squares fitting of ellipses. The Sixth International Conference in Central Europe on Computer Graphics and Visualization, Plzeò, 1998, pp. 125-132.

[9] Ramar, K. Arumugam, S.M. Sinavandam, S.N. Ganesan, L. Manimegalai, D. Quantitative fuzzy measures for threshold selection. Pattern recognition Letters, 21 2000, 1-7. 